\title{
Initial Technology Assessment for the Large-Aperture UV-Optical- Infrared (LUVOIR) Mission Concept Study
}

Matthew R. Bolcar*a, Lee Feinberga ${ }^{\mathrm{a}}$, Kevin France ${ }^{\mathrm{b}}$, Bernard J. Rauscher ${ }^{\mathrm{a}}$, David Redding ${ }^{\mathrm{c}}$, David Schiminovich $^{\mathrm{d}}$

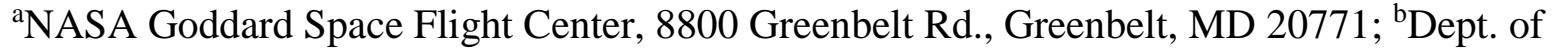
Astrophysical and Planetary Sciences, University of Colorado, UCB 600, Boulder, CO 80309; ' Jet Propulsion Laboratory, 4800 Oak Grove Dr., Pasadena, CA 91109; ${ }^{\mathrm{d} D e p t . ~ o f ~ A s t r o n o m y, ~ C o l u m b i a ~}$ University, Broadway and $116^{\text {th }}$ St., New York, NY 10027;

\begin{abstract}
The NASA Astrophysics Division's 30-Year Roadmap prioritized a future large-aperture space telescope operating in the ultra-violet/optical/infrared wavelength regime. The Association of Universities for Research in Astronomy envisioned a similar observatory, the High Definition Space Telescope. And a multi-institution group also studied the Advanced Technology Large Aperture Space Telescope. In all three cases, a broad science case is outlined, combining general astrophysics with the search for biosignatures via direct-imaging and spectroscopic characterization of habitable exoplanets. We present an initial technology assessment that enables such an observatory that is currently being studied for the 2020 Decadal Survey by the Large UV/Optical/Infrared (LUVOIR) surveyor Science and Technology Definition Team. We present here the technology prioritization for the 2016 technology cycle and define the required technology capabilities and current state-of-the-art performance. Current, planned, and recommended technology development efforts are also reported.
\end{abstract}

Keywords: large space telescopes, technology development, coronagraphy, stable systems, detectors, starshades, mirror coatings

\section{INTRODUCTION}

In early 2016, the NASA Astrophysics Division announced the establishment of four large mission concept studies to prepare for the National Academies of Science (NAS) 2020 Decadal Survey. The four missions are: The Large UltraViolet/Optical/Infrared (LUVOIR) Surveyor, the Habitable Exoplanet Imager (HabEx), the Far-Infrared Surveyor (FIRS), and the X-Ray Surveyor (XRS). The LUVOIR Science and Technology Definition Team (STDT) ${ }^{1}$, aided by the LUVOIR Study Office and Technology Working Group (TWG) and leveraging earlier studies including the High Definition Space Telescope (HDST) ${ }^{2}$ and the Advanced Technology Large Aperture Space Telescope (ATLAST) ${ }^{3,4}$, has begun to define a space telescope mission capable of the most ambitious and compelling science performance. The LUVOIR science objectives include discovery and characterization of earth-like planets around other stars, high resolution imaging reaching to individual stars in neighboring galaxies and to star-forming regions in the most distant galaxies, and spectroscopy capable of mapping tenuous inter- and intra-galactic gas flows, among many other capabilities. The LUVOIR STDT has begun to define the technologies needed to accomplish these objectives, and identified several technology gaps that require timely investment in order to prepare the LUVOIR mission concept study for review by the 2020 NAS Decadal Survey.

While a full, detailed science program for the LUVOIR mission will take some time to develop, it is certain that this program will include direct imaging and spectroscopy of planets orbiting other stars, reaching into the "habitable zone" of those stars to find candidate exoEarths. The most efficient way to accomplish this is using coronagraphic instruments behind a large aperture telescope. Coronagraphs work by suppressing the light from a distant star to a very high degree, while passing the light from planets orbiting that star. The starlight suppression ratio or "contrast" needed for imaging an exoEarth is $1: 10^{-10}$, within a small angle of $2-4$ times the diffraction-limited resolution of the telescope. Achieving this contrast performance is complicated for LUVOIR by the diffraction effects of the obscurations and segment gaps that come with the use of a large segmented telescope. Coronagraph technology now under development for the Wide-field Infrared Survey Telescope (WFIRST) ${ }^{5}$ mission shows a path forward, but more work is needed to address LUVOIRspecific challenges. Our first technology priority, discussed in Section 2, is therefore to design coronagraphs capable of the target performance with a segmented aperture; and to demonstrate them in laboratory measurements.

*matthew.bolcar@nasa.gov; phone: 1-301-286-5237; fax 1-301-286-0204; https://www.nasa.gov/goddard 
Coronagraph performance is critically dependent on the stability of the optics in the telescope. Contrast of $10^{-10}$ requires that changes in the optical wavefront error be kept below 10 picometers RMS over the time interval between coronagraph wavefront control updates ${ }^{6}$. This will require unprecedented stability, as well as exceptional quality, of the telescope optics. Our second technology priority, discussed in Section 3, is thus Ultra-Stable Opto-Mechanical Systems, to design mirror systems with associated thermal and optical sensing and controls, that are capable of picometer stability; and to demonstrate them in laboratory measurements.

It is also certain that the LUVOIR science program will include a variety of UV imaging and spectroscopic observations that will require extremely sensitive instrumentation. For low-background and time-resolved astronomy, UV detectors with photon-counting capabilities are required. The same instruments must also have high-dynamic range capabilities to allow for bright object observations. Finally, large format detectors would allow for unprecedented imaging and spectroscopic capabilities. Our third technology priority, discussed in Section 4, is thus Large Format, High Sensitivity, and High Dynamic Range (HDR) UV Detectors.

While these three technologies have received our highest prioritization and require immediate investment, we have identified additional technologies that provide important support to LUVOIR's scientific capabilities. Visible/NearInfrared detectors for exoplanet science will need extremely low noise to allow for high-yield exoplanet detection and characterization $^{7}$; investments in this area to complement continuing development may be required in the future. A starshade provides an alternative to coronagraphy for starlight suppression, with capabilities that complement a coronagraph's efficient observations; investigation of starshades for LUVOIR will provide important options, especially if coronagraphy with segmented apertures does not meet goals. High-throughput, high-uniformity mirror coatings are needed to maximize the sensitivity of a large-aperture space telescope, significantly enhancing UV performance. Finally, improved mid-infrared detectors could enable science observations out to $\sim 5 \mu \mathrm{m}$, even with a warm telescope operated at "room" temperature 8 , for exoplanet transit spectroscopy and other scientific objectives. As LUVOIR's science program continues to develop, and as higher-priority gaps begin to narrow, we expect future investments will be needed to develop these important technologies.

Figure 1 shows the prioritized list of technologies identified by the LUVOIR TWG. In the following sections, we present our highest technology priorities, describe the gap in capability, and comment on potential development paths that should be initiated immediately in the 2016 technology cycle. As part of the technology prioritization, we assess a given technology's difficulty and urgency. By difficulty, we refer to the relative challenge associated with closing the technology gap. By urgency, we refer to how soon significant investment is needed in order to mature the technology to a credible level by the 2020 Decadal Survey, as well as in time for a mission start in the mid-2020s.

\begin{tabular}{|l|c|c|}
\hline Technology Area & Difficulty & Urgency \\
\hline $\begin{array}{l}\text { High-Contrast Segmented-Aperture } \\
\text { Coronagraphy }\end{array}$ & CRITICAL & CRITICAL \\
\hline $\begin{array}{l}\text { Ultra-Stable Opto-mechanical Systems } \\
\text { (includes Sensing, Control, Mirrors, and Structures) }\end{array}$ & CRITICAL & CRITICAL \\
\hline $\begin{array}{l}\text { Large Format, High Sensitivity, High-Dynamic } \\
\text { Range UV Detectors }\end{array}$ & HIGH & HIGH \\
\hline Vis/NIR Exoplanet Detectors & HIGH & MED \\
\hline Starshade & HIGH & MED \\
\hline Mirror Coatings & MED & MED \\
\hline MIR (3-5 $\mu \mathrm{m}$ ) Detectors & LOW & LOW \\
\hline
\end{tabular}

Figure 1 - LUVOIR technology priorities. 


\section{HIGH-CONTRAST SEGMENTED-APERTURE CORONAGRAPHY}

Priority: 1

Difficulty: CRITICAL

No coronagraph technique has been demonstrated at the necessary performance level with a UV-compatible segmented aperture system. Wavefront sensing systems have not been demonstrated to close the loop fast enough to provide margin against telescope stability requirements.

Urgency: CRITICAL

The challenging nature of the problem requires a long-term development effort, during which progress will drive, and be driven by, architecture decisions throughout the LUVOIR system.

\subsection{Discussion}

One of the primary science goals of LUVOIR is to detect and characterize habitable exoplanets around nearby stars. To achieve exoplanet yields large enough to enable a statistical study of habitability requires a large aperture telescope, likely greater than 8 meters in diameter ${ }^{7}$. Such an aperture must be segmented in order to fit into current and planned launch vehicle fairings. Therefore, any coronagraphic instrument that is used to perform the exoplanet survey and characterization must be compatible with a segmented aperture telescope.

Recent achievements in the development of the WFIRST coronagraphic instrument have dispelled the widely-held belief that high-contrast imaging with a coronagraph could only be performed with a monolithic, unobscured, off-axis telescope ${ }^{9}$. WFIRST coronagraphs have achieved $\sim 10^{-8}$ contrast at an inner working angle (IWA) of $3 \lambda / \mathrm{D}$ over a $10 \%$ bandpass with an obscured monolithic aperture, in part by using the coronagraph's internal deformable mirrors (DMs) to compensate for the additional diffraction caused by the aperture obscurations. However, modeling indicates that current WFIRST coronagraph designs will achieve Airy throughputs of less than $5 \%{ }^{10}$, defined as the fraction of photons in the full-width-half-maximum (FWHM) of the planet point-spread function (PSF) divided by the number of planet photons entering the telescope aperture. These results are specific to WFIRST. Improvement is needed to meet LUVOIR performance requirements using LUVOIR candidate apertures.

Table 1 shows what we currently believe to be the performance requirements for a segmented-aperture coronagraph, including an associated low-order wavefront sensing and control (LOWFSC) system. Table 1 also presents our assessment of the current state of the art, which is embodied by the WFIRST coronagraph instrument candidates.

Table 1 - Performance goals and current state-of-the-art for a high-contrast, segmented-aperture coronagraph instrument.

\begin{tabular}{|l|l|l|}
\hline Parameter & Goal & State-of-the-Art ${ }^{9,11,12}$ \\
\hline Aperture Type & Obscured, Segmented & Obscured, Monolith \\
\hline Contrast & $1 \times 10^{-10}$ & $8.54 \times 10^{-9}$ \\
\hline Inner Working Angle & $2 \lambda / \mathrm{D} @ 1 \mu \mathrm{m}$ & $3 \lambda / \mathrm{D} @ 550 \mathrm{~nm}$ \\
\hline Bandpass (Instantaneous) & $10-20 \%$ & $10 \%$ \\
\hline Bandpass (Total) & $400 \mathrm{~nm}-1.8 \mu \mathrm{m}$ & $523 \mathrm{~nm}-578 \mathrm{~nm}$ \\
\hline Throughput & $>10 \%$ & $<5 \%$ \\
\hline LOWFSC Controllable Modes & Tip, tilt, Z4-Z11 & Tip, tilt \\
\hline LOWFSC Speed & $>1 \mathrm{kHz}$ & $\sim 1 \mathrm{kHz}$ \\
\hline LOWSFC Accuracy & $<10 \mathrm{pm}$ RMS & $<0.5$ mas RMS per axis \\
\hline Post-processing Contrast Gain & $>10 \times$ & $3 \times$ \\
\hline
\end{tabular}

Current investments in WFIRST coronagraph development, especially with respect to the LOWFSC system and postprocessing can be directly leveraged and applied to LUVOIR. Additionally, some of NASA's SAT Technology Development for Exoplanet Missions (TDEM) ${ }^{13}$ portfolio has been awarded to the development of segmented-aperture coronagraphs, namely the Visible Nulling Coronagraph (VNC) ${ }^{14}$ and the Vector Vortex Coronagraph (VVC) ${ }^{15}$.

The Exoplanet Exploration (ExEP) program office has also funded a Segmented Coronagraph Design \& Analysis (SCDA) study $^{16}$ to model coronagraph performance with an array of segmented aperture architectures. Multiple techniques are being studied, including: Phase-Induced Amplitude Apodization Complex-Mask Coronagraphs (PIAA$\mathrm{CMC})^{17}$, Apodized Pupil Lyot Coronagraphs (APLC) ${ }^{18}$, Vortex Hybrid Lyot Coronagraphs, and the VNC. 


\subsection{Recommendation}

In order to achieve the challenging goals outlined in Table 1, we recommend that the SCDA study continue investigating new coronagraph techniques and designs, as well as improve on the fidelity of the modeling being performed in this first-round effort (i.e. include dynamics, relevant wavefront error terms, etc.). Most importantly, viable segmented aperture coronagraph candidates must be demonstrated with a UV-compatible segmented aperture testbed prior to 2020 in order to show credibility at the Decadal Survey. Such a demonstration should include the LOWFSC system necessary to correct for relevant instabilities.

\section{ULTRA-STABLE OPTO-MECHANICAL SYSTEMS}

Priority: 2

Difficulty: CRITICAL

The wavefront error stability requirement is unprecedented, and requires a systems-level approach that includes all aspects of the LUVOIR architecture. LUVOIR science goals are critically coupled to the ability of the opto-mechanical system to deliver a wavefront stable at the picometer level.

Urgency: CRITICAL

The systems-level nature of this technology necessitates immediate investment so that decisions that impact all aspects of the architecture can be made early to reduce cost and risk. The challenging nature of the problem will also require a long-term development effort.

\subsection{Discussion}

The telescope static wavefront error performance will likely be set by the UV and visible imaging science requirements, nominally to achieve diffraction limited performance at the short end of the visible spectrum, after correction by segment rigid-body actuators (and segment figure actuators if so equipped). Also important will be ensuring that mid-spatialfrequency wavefront errors are within the correction range of deformable mirrors in the coronagraphic imager. Primary mirror segments with post-control wavefront error contributions from uncorrected surface figure in the range of $10 \mathrm{~nm}$ RMS will likely be required.

The most critical requirement on the opto-mechanical system, and the most challenging, is that of wavefront error stability: to enable the high-contrast imaging with a coronagraphic instrument, the wavefront error must be stable on the order of $10 \mathrm{pm}$ per wavefront control step ${ }^{6}$.

As mentioned earlier, the aperture sizes that are necessary for LUVOIR's science objectives imply that the primary mirror will likely need to be segmented, similar to that of the James Webb Space Telescope (JWST). Thus, primary mirror segment phasing becomes a key factor in maintaining the wavefront error stability. Stability of the secondary mirror position relative to the primary mirror is also important although tolerances are $\sim 100 \times$ looser in positioning, based on preliminary modeling.

Also similar to JWST, LUVOIR will likely be operated in a halo orbit about the Sun-Earth L2 Lagrange point, behind a large sunshade, which will passively provide a high level of stability. This sunshade will provide a low ambient temperature, a large cold bias against which heaters can be operated for efficient thermal control ${ }^{19}$.

Table 2 shows what we believe are the performance requirements for the LUVOIR opto-mechanical system, as well as our assessment of the current state-of-the-art.

Table 2 - Performance goals and current state-of-the-art for the ultra-stable opto-mechanical system.

\begin{tabular}{|l|l|l|}
\hline Parameter & Goal & State-of-the-Art ${ }^{20,21}$ \\
\hline Mirror Surface Figure Error: & $<5 \mathrm{~nm}$ RMS & $<7 \mathrm{~nm}$ RMS \\
\hline Wavefront Error Stability & $<10 \mathrm{pm} /$ control step & $\sim 50 \mathrm{~nm} /$ control step \\
\hline
\end{tabular}

In order to achieve picometer stability per control step, two approaches can be taken. First, in the scenario of a slow wavefront control system, the system must be made to be extraordinarily stable such that the wavefront error does not change over long periods of time (10s of minutes, for example). Conversely, if the wavefront control system can operate quickly (i.e. a few $\mathrm{Hz}$ ), then the system stability can be relaxed, as the control system will be able to keep up with dynamic and thermal drifts. 
In either scenario, there are three common components that enable the opto-mechanical stability: the ability to sense the wavefront drift, the ability to correct the wavefront drift, and the mirror and structure that must be held stable.

\section{Sensing Technologies:}

There are two general classes to sensor technologies: those that use the light from objects being observed to sense the wavefront, such as image-based techniques, or the Zernike wavefront sensor (ZWFS) employed by the WFIRST coronagraph's LOWFSC system ${ }^{11}$; and external metrology systems such as laser trusses or capacitive edge sensors ${ }^{22,23}$. The former are an example of slower sensing technologies since they depend on the relatively faint objects being observed, and thus must integrate for long times to build up enough signal. In contrast, external metrology systems can be made to be almost arbitrarily fast, but at the expense of added system complexity.

Each sensor has heritage in systems used today. Laser trusses, which might be an ideal candidate for maintaining primary-to-secondary mirror alignment, have heritage in the development of flight systems for the Space Interferometry Mission (SIM) ${ }^{24}$; shorter-range sensors used in the commercial microlithography industry have achieved 10 pm RMS sensitivity ${ }^{25}$. Capacitive edge sensors have been baselined for the Thirty Meter Telescope (TMT) as part of the mirror co-phasing system ${ }^{26}$. Image-based wavefront sensing techniques are baselined as the primary means to commission and maintain alignment of the JWST ${ }^{27}$. And as mentioned earlier, ZWFSs are currently being developed for the WFIRST coronagraph instrument's LOWFSC system ${ }^{11}$. It is likely that a combination of these techniques will be needed for LUVOIR, although gains in sensitivity and sensing speed are required across the board.

\section{Control Technologies:}

Once the wavefront drift has been sensed, it must be controlled, and again there are several options. Deformable mirrors (DMs), both macro-scale ${ }^{28}$ and micro-electromechanical systems (MEMS) ${ }^{29}$, allow for wavefront correction at several stages in the optical system, including within the coronagraph instrument. Most DMs have a continuous facesheet backed by actuators to control the surface. MEMS DMs also exist in segmented formats ${ }^{30}$, where each segment can be actuated in piston, tip, and tilt degrees of freedom (DOF).

Additionally, the primary mirror segments themselves can be actuated. Rigid-body hexapods can control each segment in 6 DOFs, warping harnesses can control low-order segment surface figure modes, and embedded surface figure actuators (SFAs) ${ }^{31}$ can be used to control higher-order segment surface figure error.

A key component of all of these control technologies is the electronics used to address the actuators. LUVOIR will require electronics with the precision, multiplexing, and robustness needed to deliver picometer controllability at high speeds.

\section{Stable Structures and Mirrors:}

Even with a high-speed sensing and control architecture, there will be some period of time between control steps when the wavefront will drift. There will also be some spatial and temporal frequency components of the drift that will be outside of the sensing and control bandpass, and will therefore remain uncontrolled. Thus, mirror and structure stability is critical and will ultimately provide margin against the sensing and control architecture.

For mirrors, ULE®, Zerodur ${ }^{\circledR}$, and silicon carbide $(\mathrm{SiC})$ are all possible materials and modeling has shown what type of thermal controls are needed for each ${ }^{32}$. For room temperature operation, the thermal control of the mirrors needs to be 1 $\mathrm{mK}$ for ULE® and Zerodur ${ }^{\circledR}$, and $\sim 0.1 \mathrm{mK}$ for SiC. At cold temperatures of $140 \mathrm{~K}$ or below, the coefficient of thermal expansion (CTE) of $\mathrm{SiC}$ drops to levels below that of ULE®, and the thermal control requirements rise. Technologies for achieving this level of thermal control and demonstrations of mirror stability that potentially include integrated active controls are still needed, at whatever temperature is chosen for operation of the telescope.

For structures, the state-of-the-art is high stability composites, such as those used on JWST, Chandra, and the Hubble Space Telescope (HST). Key future needs are lower coefficient of moisture expansion, better CTEs around a very small temperature window, and an understanding of small lurches due to internal stress relaxation or environmental changes. Other backplane materials, including low-CTE SiC are also of interest.

Beyond just the mirror and structure materials, picometer stability requires the consideration of every bond, joint, and material in the assembly, including epoxies, interfaces, flexures, fasteners, and actuators. Lurch, linearity, and the time scale of material change must be thoroughly understood to assure that the $10 \mathrm{pm}$ budget can be met within the sensing and control bandpass. 


\subsection{Recommendation}

The systems-level nature of the wavefront stability problem requires a systems-level approach to solving the problem. Mirrors, structures, sensors, actuators, materials, modeling, and the overall architecture must be developed together, as a system. Most importantly, this development will require substantial participation of industry, as that is where much of the expertise in these areas resides. We recommend that NASA sponsor design and development activities to demonstrate closed-loop picometer-class wavefront performance for segmented apertures, and the ability to verify that stability on the ground. Furthermore, it would be advantageous to pursue concurrent, competitive development of picometer component sensing and control technologies along with architecture feasibility demonstrations and assessments.

\section{LARGE FORMAT, HIGH SENSITIVITY, HIGH DYNAMIC RANGE ULTRAVIOLET DETECTORS}

Priority: 3

Difficulty: HIGH

Multiple technology candidates exist, but each require improvements in different performance areas. Parallel development paths are required until an acceptable candidate is realized.

Urgency: HIGH

UV sensitivity is critical to enabling LUVOIR's first-launch science objectives. However, we recognize that serviceability enables future upgrade paths for both detector and instrument capabilities.

\subsection{Discussion}

LUVOIR's aperture size and unprecedented stability will naturally provide gains in sensitivity and image quality at all wavelengths. In order to enable new science in the UV, however, additional improvements are required in UV detector sensitivity (quantum efficiency (QE) and noise performance), dynamic range, and pixel count.

There are three basic technologies considered for UV detectors. Micro-channel plates (MCPs) ${ }^{33}$ with "solar-blind" photocathodes, low dark current, and zero read noise that are fairly mature and have rich flight heritage. However, MCPs have limited dynamic range and issues with long-term "gain-sag" (burn-in of regions exposed to prolonged high illumination levels). Detector technologies such as electron-multiplying charge-coupled devices (EMCCDs) ${ }^{34}$ devices and scientific complementary metal-oxide-semiconductor (sCMOS) ${ }^{35}$ offer new options in terms of format and dynamic range, but are less mature, less solar-blind, and have yet to be evaluated, or optimized, for the harsh radiation environment. Once EMCCDs and sCMOS detectors have been demonstrated to meet the fundamental noise requirements for science observations and to be radiation hard, then they could be $\delta$-doped to improve UV performance. It is also noteworthy that evaluation and radiation hardening of EMCCD and sCMOS detectors directly benefits our fourth priority technology: Vis/IR Detectors for Exoplanet Science.

A principal goal for all technologies is the development of a large-format detector with high pixel count to allow for wide-field imaging and multi-object spectroscopy in the UV. All technologies need additional development to achieve these high fill-factor focal planes with a large pixel count, while maintaining other capability requirements. Curved detectors that have the potential to simplify relay optics and reduce the number of reflections for wide-field of view performance should also be considered.

Table 3 shows what we believe to be the fundamental performance requirements for UV detectors for LUVOIR, as well as our assessment of the current state-of-the-art for each of the technology candidates. Note, due to the fundamentally different architecture between MCPs and EMCCD/sCMOS devices, we use the term "resol" to refer to a spatial resolution element for MCPs, and a two-pixel width for EMCCDs and sCMOS. 
Table 3 - Performance goals and current state-of-the-art for candidate UV detector technologies.

\begin{tabular}{|c|c|c|c|}
\hline Parameter: & Goal: & State-of-the-Art: ${ }^{33-37}$ & \\
\hline \multirow{3}{*}{ Operational Bandpass } & \multirow{3}{*}{$90 \mathrm{~nm}-400 \mathrm{~nm}$} & $90 \mathrm{~nm}-300 \mathrm{~nm} *$ & $\mathrm{MCP}$ \\
\hline & & $90 \mathrm{~nm}-400 \mathrm{~nm}$ & EMCCD \\
\hline & & TBD & sCMOS \\
\hline \multirow{3}{*}{ Read Noise } & \multirow{3}{*}{0} & 0 & $\mathrm{MCP}$ \\
\hline & & N/A for multi. mode & EMCCD \\
\hline & & $0.8-1.0 \mathrm{e}^{-}$ & sCMOS \\
\hline \multirow{3}{*}{ Dark Current } & \multirow{3}{*}{0} & 0 & MCP \\
\hline & & $>0.005 \mathrm{e}^{-} / \mathrm{resol} / \mathrm{hr}$ & EMCCD \\
\hline & & $>0.005 \mathrm{e}^{-} / \mathrm{resol} / \mathrm{hr}$ & sCMOS \\
\hline \multirow{3}{*}{ Spurious Count Rate } & \multirow{3}{*}{$\leq 0.05$ counts $/ \mathrm{cm}^{2} / \mathrm{s}$} & 0.05 counts $/ \mathrm{cm}^{2} / \mathrm{s}$ & MCP \\
\hline & & TBD & EMCCD \\
\hline & & TBD & sCMOS \\
\hline \multirow{3}{*}{ Quantum Efficiency (Peak) } & \multirow{3}{*}{$\begin{array}{l}75 \% \\
\text { (Far UV - Near UV) }\end{array}$} & $45-20 \%$ FUV - NUV & MCP \\
\hline & & $30-50 \%$ FUV - NUV & EMCCD \\
\hline & & TBD & sCMOS \\
\hline \multirow{3}{*}{ Resol Size } & \multirow{3}{*}{$\leq 10 \mu \mathrm{m}$} & $20 \mu \mathrm{m}$ & $\mathrm{MCP}$ \\
\hline & & $20 \mu \mathrm{m}$ & EMCCD \\
\hline & & $10-20 \mu \mathrm{m}$ & sCMOS \\
\hline \multirow{3}{*}{$\begin{array}{l}\text { Dynamic Range } \\
\text { (Max. Count Rate) }\end{array}$} & \multirow{3}{*}{$\begin{array}{l}\geq 10^{4} \mathrm{~Hz} / \mathrm{resol} \\
\text { (as needed) }\end{array}$} & $\begin{array}{l}40 \mathrm{~Hz} / \text { resol } \\
5 \mathrm{MHz} \text { global }\end{array}$ & $\mathrm{MCP}$ \\
\hline & & Readout dependent & EMCCD \\
\hline & & $10^{5} \mathrm{~Hz} /$ resol & sCMOS \\
\hline \multirow{3}{*}{ Time Resolution } & \multirow{3}{*}{$\begin{array}{l}\leq 100 \mathrm{~ms} \\
\text { (as needed) }\end{array}$} & $<<1 \mathrm{~ms}$ & $\mathrm{MCP}$ \\
\hline & & $<10 \mathrm{~ms}$ & EMCCD \\
\hline & & $<10 \mathrm{~ms}$ & sCMOS \\
\hline \multirow{3}{*}{ Format } & \multirow{3}{*}{$\begin{array}{l}\geq 8-16 \mathrm{k} \text { pixels per side } \\
\text { with high fill factor }\end{array}$} & $8 \mathrm{k} \times 8 \mathrm{k}$ & $\mathrm{MCP}$ \\
\hline & & $3.5 \mathrm{k} \times 3.5 \mathrm{k}$ & EMCCD \\
\hline & & $3.5 \mathrm{k} \times 3.5 \mathrm{k}$ & sCMOS \\
\hline \multirow{3}{*}{ Radiation Tolerance } & \multirow{3}{*}{ Good } & Good & $\mathrm{MCP}$ \\
\hline & & Fair & EMCCD \\
\hline & & Good & sCMOS \\
\hline
\end{tabular}

Laboratory and suborbital flight testing programs are ongoing for MCPs with cross-strip, delay-line, and applicationspecific integrated circuit (ASIC) readouts to improve dynamic range. These activities are currently funded by the SAT and APRA programs, and also receive support for flight testing from sub-orbital programs. Delta-doped EMCCDs are also receiving funding from the SAT, APRA, and suborbital flight programs, but require additional demonstrations of UV photon-counting capability while remaining "solar blind" (insensitive above wavelengths of $400 \mathrm{~nm}$ ) and able to maintain performance in the expected radiation environment.

\subsection{Recommendation}

Technology maturation activities may be accelerated with direct technology-only rocket/balloon missions and/or laboratory demonstrations. The time and effort expended on optimizing the science instrumentation may be minimized resulting in a faster technology development or launch timeframe. A dedicated program-supported laboratory and suborbital technology development program could also accomplish this goal. The radiation hardness of EMCCDs must first be evaluated and addressed before significant resources are applied to $\delta$-doping for UV enhancement. It is strongly recommended that SCMOS devices be evaluated by independent parties for noise performance and radiation hardness as a high priority, especially since these devices may also serve as visible-band detectors for LUVOIR's exoplanet science mission. 


\section{ADDITIONAL TECHNOLOGY PRIORITIES}

The additional technologies identified in Figure 1 have also been prioritized by the LUVOIR TWG for development. However, their difficulty or urgency does not require extraordinary measures beyond those investments that are already being made, or will likely be made available through existing funding programs in 2016. For completeness, we briefly comment on each here. As higher-priority technology gaps begin to narrow, and as the 2020 Decadal Survey approaches, it is certain these technologies will rise in priority.

\subsection{Vis/NIR Detectors for Exoplanet Science}

Visible and near-infrared detectors with extremely low noise (read noise, dark current, clock-induced charge) are necessary to achieve the highest exoEarth detection and characterization yields with LUVOIR. The exact performance requirements depend on what type of detector is used (single-photon vs. energy resolving) and what type of instrument is used for detecting the exoplanet (wide-band imager vs. integral field spectrograph). Generally speaking, detectors with dark currents less than 0.0010 counts / pixel / second and read noise less than $1 \mathrm{e}^{-} /$pixel are needed. These detectors must also be capable of maintaining their performance in the radiation environment at the Sun-Earth L2 point for the duration of a 5-year primary science mission.

Several candidate technologies exist, including EMCCDs ${ }^{34}$ and $\operatorname{sCMOS}^{35}$ devices in the visible band and $\mathrm{HgCdTe}$ avalanching photodiode (APD) ${ }^{36}$ arrays in the NIR. Energy resolving detectors such as microwave kinetic inductance detectors (MKIDs) and Transition Edge Sensors (TES) ${ }^{37}$ are also candidates at all wavelengths, but require cryogenic operation, which may have other system-level impacts to stability. For now, we will closely monitor the progress of EMCCD evaluation for WFIRST, and revise our recommendation as more is learned about their performance and radiation hardness. We strongly advise that additional detector technologies, especially sCMOS, be evaluated against the performance requirements as soon as possible to help determine their suitability for LUVOIR. As mentioned earlier, characterization of EMCCD and SCMOS detectors against the science and radiation requirements would benefit both the Vis/IR Detectors for Exoplanet Science and Large Format, High Sensitivity, High Dynamic Range UV Detector technology priorities.

\subsection{Starshade}

Starshades ${ }^{38}$ offer an alternative means of starlight suppression over a high-contrast segmented aperture coronagraph. While starshades do not require the same level of wavefront stability as a coronagraph, they are another entire spacecraft that must be engineered, fabricated, launched, and then precisely flown in formation with LUVOIR. There is also evidence that if the starshade is used for the survey portion of the exoplanet science campaign, the exoEarth yields would not be as high as those achieved by an internal coronagraph ${ }^{39}$.

Still, the starshade technology should be pursued for two reasons. First, it offers a risk reduction path should a suitable segmented aperture coronagraph not be developed, or should picometer-level wavefront stability not be achievable. Secondly, starshades have an advantage over coronagraphs in exoplanet characterization, especially in the infrared. Since a coronagraph's inner working angle scales with wavelength, as the wavelength increases, the coronagraph must work closer to the stellar core for the same planet, where suppression becomes more challenging. Starshades are less sensitive to this issue.

We note that NASA's Exoplanet Exploration Program Office has recently chartered the starshade working group $(\mathrm{SSWG})^{40}$ to develop "a technical concept and risk reduction plan for the technology validation of starshades." For now, it is sufficient for the LUVOIR community to provide inputs to the SSWG and track its progress.

\subsection{Mirror Coatings}

To maximize the sensitivity of LUVOIR's large aperture, additional development of high-performance mirror coatings is needed. It is a given that a reflective aluminum surface will be needed on at least the primary and secondary mirrors to enable science observations in the UV down to $\sim 90 \mathrm{~nm}$. To prevent oxidation of the aluminum layer, a thin dielectric overcoat, such as $\mathrm{MgF} 2, \mathrm{LiF}$, or $\mathrm{AlF} 3$, is typically deposited on top of the aluminum. The type of material and deposition process determines the final reflectivity, uniformity, and durability of the coating.

All metallic coatings also induce a polarization change on the light that is reflected, an effect that is dependent on the angle-of-incidence, and is usually increased by the dielectric overcoat. The net effect is polarization-induced aberration, whereby each polarization state of the incident light "sees" a different wavefront error upon propagation through the 
system. This presents a challenge to high-contrast imaging with a coronagraph, since only a single polarization state can be sensed and controlled at a time, requiring serial observations in each polarization state, or dual coronagraph instruments operating in parallel ${ }^{41}$. Cross-polarization leakage also creates an incoherent point-spread function (PSF) at the focal plane that may limit the achievable contrast floor. Coronagraph demonstrations as part of our highest-priority technology gap should be performed with a UV-compatible telescope to help retire this risk as soon as possible.

Investments in improving deposition processes is needed, as well as basic materials science research to understand if fundamental improvements can be made in coating performance, specifically with respect to reducing polarizationinduced aberration.

\subsection{Mid-Infrared (3-5 $\mu \mathrm{m})$ Detectors:}

While it is anticipated that LUVOIR will be a "warm" telescope operating at or near room temperature, it is still possible that some observations in the mid-infrared may be possible, especially if the option for a cold instrument within the instrument suite is allowed ${ }^{8}$. Obviously, such observations will be limited by the telescope's thermal background. Still, we recognize the potential need for improved sensitivity for MIR detectors, either for general astrophysics or exoplanet science.

\section{CONCLUSION}

We have reported on the initial technology assessment and prioritization conducted by the LUVOIR STDT, Study Office, and TWG. Seven technologies have been identified, and three have been prioritized for immediate funding in 2016: high-contrast segmented-aperture coronagraphy; ultra-stable opto-mechanical systems; and large format, highsensitivity, high-dynamic range UV detectors. Additional technologies, including Vis/IR detectors for exoplanet

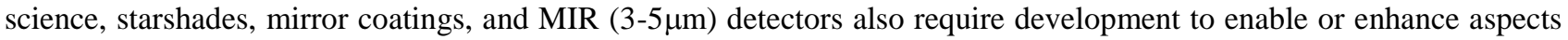
of the LUVOIR primary science mission. We fully expect that as additional definition of the LUVOIR mission and its science objectives is realized, and as technology development progresses, this technology list will need to be reprioritized in the future.

\section{ACKNOWLEDGEMENTS}

The authors would like to thank the LUVOIR STDT members and Technology Working Group members that contributed to discussions regarding the technology prioritization, including: Jon Arenberg, Kunjithapatham Balasubramanian, Alberto Conti, Jeanette Domber, Marc Ferrari, Jay Gallagher, Walter Harris, Brad Peterson, Marc Postman, Laurent Pueyo, Manuel Quijada, Karl Stapelfeldt, and Chris Stark. The authors note that much of this text appeared as the LUVOIR "O1" Deliverable to the NASA Astrophysics Science Division as part of the Decadal Mission Concept Study Management Plan ${ }^{42}$.

\section{REFERENCES}

[1] "Large UV/Optical/Infrared Surveyor: Meet the Team,” 31 May 2016 (accessed 15 June 2016)

http://asd.gsfc.nasa.gov/luvoir/team/.

[2] J. Dalcanton, S. Seager, S. Aigrain, S. Battel, N. Brandt, C. Conroy, L. Feinberg, S. Gezari, O. Guyon, W. Harris, C. Hirata, J. Mather, M. Postman, D. Redding, D. Schiminovich, H. P. Stahl, and J. Tumlinson, From Cosmic Birth to Living Earths: The Future of UVOIR Space Astronomy, published by the Association of Universities for Research in Astronomy 2015 (accessed 15 June 2016), http://www.hdstvision.org/report/.

[3] H. Thronson, M. R. Bolcar, M. Clampin, J. Crooke, L. Feinberg, W. Oegerle, N. Rioux, H. P. Stahl, and K. Stapelfeldt, "A Path to a UV/Optical/IR Flagship: ATLAST and Its Predecessors," J. Astron. Telesc. Instrum. Syst., in press. 
[4] M. R. Bolcar, K. Balasubramanian, J. Crooke, L. Feinberg, M. Quijada, B. J. Rauscher, D. Redding, N. Rioux, S. Shaklan, H. P. Stahl, C. Stahle, and H. Thronson, "A Technology Gap Assessment for a Future Large-Aperture Ultraviolet-Optical-Infrared Space Telescope,” J. Astron. Telesc. Instrum. Syst., manuscript under review.

[5] WFIRST Study Office and Science Definition Team, "Wide-Field Infrared Survey Telescope - Astrophysics Focused Telescope Assets (WFIRST-AFTA) 2015 Report, 10 March 2015 (accessed 15 June 2016), http://wfirst.gsfc.nasa.gov/science/sdt_public/WFIRST-AFTA_SDT_Report_150310_Final.pdf.

[6] M. T. Stahl, S. B. Shaklan, and H. P. Stahl, "Preliminary analysis of effect of random segment errors on coronagraph performance ", Proc. SPIE 9605, Techniques and Instrumentation for Detection of Exoplanets VII, 96050P (September 24, 2015), [doi:10.1117/12.2190160].

[7] C. C. Stark, A. Roberge, A. Mandell, M. Clampin, S. D. Domagal-Goldman, M. W. McElwain, and K. R. Stapelfeldt, "Lower Limits on Aperture Size for an ExoEarth Detecting Coronagraphic Mission," ApJ, 808(149), 1-16 (2015), [doi:10.1088/0004-637X/808/2/149].

[8] M. Werner, M. Swain, G. Vasisht, X. Wang, S. Macenka, A. Mandell, S. Domagal-Goldman, J. Green, and C. Stark, "Extension of ATLAST/LUVOIR's Capabilities to $5 \mu \mathrm{m}$, or Beyond," J. Astron. Telesc. Instrum. Syst., manuscript under review. Preprint available at arXiv:1605:04367.

[9] E. Cady, B.-J. Seo, K. Balasubramanian, F. Greer, B. Gordon, N. J. Kasdin, B. Kern, A. Kuhnert, D. Marx, C. M. Prada, D. Moody, R. Muller, I. Poberezhskiy, A. J. Riggs, J. Trauger, D. Wilson, V. White, K. Yee, N. Zimmerman, and H. Zhou, "Milestone 5: Hybrid Lyot and Shaped Pupil Broadband Contrast Testbed Demonstration for WFIRSTAFTA," (accessed 15 June 2016), http://wfirst.gsfc.nasa.gov/science/sdt_public/wps/references/ WFIRST_CGI_Milestone5_Final_Report.pdf.

[10] J. Krist, B. Nemati, and B. Mennesson, "Numerical modeling of the proposed WFIRST-AFTA coronagraphs and their predicted performances," Journal of Astronomical Telescopes, Instruments, and Systems, 2(1), 011003 (2015), [doi:10.1117/1.JATIS.2.1.011003].

[11] F. Shi, K. Balasubramanian, R. Bartos, R. Hein, B. Kern, J. Krist, R. Lam, D. Moore, K. Patterson, I. Poberezhskiy, J. Shields, R. Sidick, H. Tang, T. Truong, K. Wallace, X. Wang, and D. Wilson, "Low order wavefront sensing and control for WFIRST-AFTA coronagraph," Proc. SPIE 9605, Techniques and Instrumentation for Detection of Exoplanets VII, 960509 (September 16, 2015), [doi:10.1117/12.2188775].

[12] M. Ygouf, L. Pueyo, R. Soummer, M. D. Perrin, R. van der Marel, and B. Macintosh, "Data processing and algorithm development for the WFIRST-AFTA coronagraph: reduction of noise free simulated images, analysis and spectrum extraction with reference star differential imaging," Proc. SPIE 9605, Techniques and Instrumentation for Detection of Exoplanets VII, 96050S (September 16, 2015), [doi:10.1117/12.2188669].

[13] Exoplanet Exploration Program Office: Technology: TDEM Awards, (accessed 15 June 2016), https://exoplanets.jpl.nasa.gov/exep/technology/TDEM-awards/.

[14] B. A. Hicks, R. G. Lyon, P. Petrone, I. J. Miller, M. R. Bolcar, M. Clampin, M. A. Helmbrecht, and U. Mallik, "Demonstrating broadband billion-to-one contrast with the Visible Nulling Coronagraph," Proc. SPIE 9605, Techniques and Instrumentation for Detection of Exoplanets VII, 96050K (September 11, 2015), [doi:10.1117/12.2189101].

[15] D. Mawet, E. Serabyn, D. Moody, B. Kern, A. Niessner, A. Kuhnert, D. Shemo, R. Chipman, S. McClain, and J. Trauger, "Recent results of the second generation of vector vortex coronagraphs on the high-contrast imaging testbed at JPL", Proc. SPIE 8151, Techniques and Instrumentation for Detection of Exoplanets V, 81511D (September 15, 2011), [doi:10.1117/12.896070]. 
[16] N. Siegler and S. Shaklan, "Segmented Coronagraph Design \& Analysis: Task Description," (accessed 15 June 2016), https://exoplanets.jpl.nasa.gov/system/internal_resources/details/original/210_SCDA_Summary.pdf.

[17] O. Guyon, P. M. Hinz, E. Cady, R. Belikov, and F. Martinache, "High Performance Lyot and PIAA Coronagraphy for Arbitrarily Shaped Telescope Apertures," Ap. J., 780(2), 171-188 (2013), [10.1088/0004-637x/780/2/171].

[18] M. N'Diaye, L. Pueyo, and R. Soummer, “Apodized Pupil Lyot Coronagraphs for Arbitrary Apertures IV: Reduced Inner Working Angle and Increased Robustness to Low-Order Aberrations," Ap. J., 799(2), 225-237 (2015), [10.1088/0004-637x/799/2/225].

[19] L. Feinberg, N. Rioux, M. R. Bolcar, A. Liu, O. Guyon, C. Stark, and J. Arenberg, "End-to-end Assessment of a Large Aperture Segmented Ultraviolet Optical Infrared (UVOIR) Telescope Architecture," Proc. SPIE 9904, Space Telescopes and Instrumentation 2016: Optical, Infrared, and Millimeter Wave, 9904-16 (June 26, 2016).

[20] L. A. Montagnino, “Test and Evaluation of the Hubble Space Telescope 2.4-meter Primary Mirror," Proc. SPIE 0571, Large Optics Technology, 182 (February 21, 1986), [doi:10.1117/12.950408].

[21] B. Saif, M. Bluth, P. Greenfield, W. Hack, B. Eegholm, P. Blake, R. Keski-Kuha, L. Feinberg, and J. W. Arenberg, "Measurement of large cryogenic structures using a spatially phase-shifted digital speckle pattern interferometer," Appl. Opt. 47, 737-745 (2008), [doi:10.1364/AO.47.000737].

[22] F. Zhao, "Development of high-precision laser heterodyne metrology gauges", Proc. SPIE 5634, Advanced Sensor Systems and Applications II, 247 (February 28, 2005), [doi:10.1117/12.569844].

[23] S. Buous, J. Menzies, and H. Gajjar, "SALT segmented primary mirror: commissioning capacitive edge sensing system and performance comparison with inductive sensor", Proc. SPIE 7012, Ground-based and Airborne Telescopes II, 70123G (August 27, 2008), [doi:10.1117/12.788909].

[24] S. B. Shaklan, L. F. Marchen, F. Zhao, R. D. Peters, T. Ho, and B. Holmes, "Metrology system for the Terrestrial Planet Finder Coronagraph", Proc. SPIE 5528, Space Systems Engineering and Optical Alignment Mechanisms, 22 (September 30, 2004), [doi:10.1117/12.559998].

[25] D. Redding, “Active Optics and Large Mirror Telescopes,” ESA Space Optics Instrument Technologies Course, Poltu Quatu, Sardinia, Italy (9 May 2016).

[26] T. Mast, G. Chanan, J. Nelson, R. Minor, and R. Jared, "Edge sensor design for the TMT", Proc. SPIE 6267, Ground-based and Airborne Telescopes, 62672S (June 23, 2006), [doi:10.1117/12.672028].

[27] B. H. Dean, D. L. Aronstein, J. S. Smith, R. Shiri, and D. S. Acton, "Phase retrieval algorithm for JWST Flight and Testbed Telescope", Proc. SPIE 6265, Space Telescopes and Instrumentation I: Optical, Infrared, and Millimeter, 626511 (June 13, 2006), [doi:10.1117/12.673569].

[28] A. Wirth, J. Cavaco, T. Bruno, and K. Ezzo, "Deformable mirror technologies at AOA Xinetics ", Proc. SPIE 8780, High-Power, High-Energy, and High-Intensity Laser Technology; and Research Using Extreme Light: Entering New Frontiers with Petawatt-Class Lasers, 87800M (May 7, 2013), [doi:10.1117/12.2018031].

[29] A. Norton, J. W. Evans, D. Gavel, D. Palmer, B. Macintosh, K. Morzinski, and S. Cornelissen, "Preliminary characterization of Boston Micromachines' 4096-actuator deformable mirror", Proc. SPIE 7209, MEMS Adaptive Optics III, 72090I (February 23, 2009), [doi:10.1117/12.811681]. 
[30] M. A. Helmbrecht, M. He, C. J. Kempf, and M. Besse, "MEMS DM development at Iris AO, Inc.", Proc. SPIE 7931, MEMS Adaptive Optics V, 793108 (February 14, 2011), [doi:10.1117/12.876186].

[31] G. Hickey, T. Barbee, M. Ealey, and D. Redding, "Actuated hybrid mirrors for space telescopes", Proc. SPIE 7731, Space Telescopes and Instrumentation 2010: Optical, Infrared, and Millimeter Wave, 773120 (August 5, 2010), [doi:10.1117/12.858217].

[32] M. J. Eisenhower, L. M. Cohen, L. D. Feinberg, G. W. Matthews, J. A. Nissen, S. C. Park, and H. L. Peabody, "ATLAST ULE mirror segment performance analytical predictions based on thermally induced distortions ", Proc. SPIE 9602, UV/Optical/IR Space Telescopes and Instruments: Innovative Technologies and Concepts VII, 96020A (September 22, 2015), [doi:10.1117/12.2188008].

[33] M. Pfeifer, J. Barnstedt, C. Bauer, S. Diebold, S. Hermanutz, C. Kalkuhl, N. Kappelmann, S. Lochner, T. Schanz, M. Schmelling, and K. Werner, "Low-power readout electronics for micro channel plate detectors with cross-strip anodes ", Proc. SPIE 8443, Space Telescopes and Instrumentation 2012: Ultraviolet to Gamma Ray, 844320 (September 7, 2012), [doi:10.1117/12.925137].

[34] D. Burt, J. Endicott, P. Jerram, P. Pool, D. Morris, A. Hussain, and P. Ezra, "Improving radiation tolerance in e2v CCD sensors," Proc. SPIE 7439, Astronomical and Space Optical Systems, 743902 (August 26, 2009); [doi:10.1117/12.825273].

[35] See, for example, ANDOR sCMOS cameras, (accessed 15 June 2016), http://www.andor.com/scientificcameras/neo-and-zyla-scmos-cameras.

[36] M. L. Bryan, G. Chapman, D. N. B. Hall, M. D. Jack, S. M. Jacobson, and J. Wehner, "Investigation of linear-mode photon-counting HgCdTe APDs for astronomical observations ", Proc. SPIE 8453, High Energy, Optical, and Infrared Detectors for Astronomy V, 84532F (September 25, 2012), [doi:10.1117/12.927268].

[37] B. J. Rauscher, M. R. Bolcar, M. Clampin, S. D. Domagal-Goldman, M. W. McElwain, S. H. Moseley, C. Stahle, C. C. Stark, and H. A. Thronson, "ATLAST detector needs for direct spectroscopic biosignature characterization in the visible and near-IR ", Proc. SPIE 9602, UV/Optical/IR Space Telescopes and Instruments: Innovative Technologies and Concepts VII, 96020D (September 22, 2015), [doi:10.1117/12.2186554].

[38] S. Seager, et al., "Exo-S: Starshade Probe-Class Exoplanet Direct Imaging Mission Concept: Final Report" March, 2015, (accessed 8 June 2016) http://exep.jpl.nasa.gov/stdt/Exo-S Starshade Probe Class Final

Report 150312 URS250118.pdf.

[39] C. C. Stark, S. Shaklan, D. Lisman, E. Cady, D. Savransky, A. Roberge, and A. M. Mandell, "Maximized exoEarth candidate yields for starshades", J. Astron. Telesc. Instrum. Syst. 2(4), 041204 (Jun 14, 2016), [doi:10.1117/1.JATIS.2.4.041204].

[40] Exoplanet Exploration Program Office: StarShade Readiness Working Group (SSWG), (accessed 15 June 2016), http://exep.jpl.nasa.gov/sswg/.

[41] K. Balasubramanian, S. Shaklan, A. Give'on, E. Cady, and L. Marchen, "Deep UV to NIR space telescopes and exoplanet coronagraphs: a trade study on throughput, polarization, mirror coating options and requirements", Proc. SPIE 8151, Techniques and Instrumentation for Detection of Exoplanets V, 81511G (September 15, 2011), [doi:10.1117/12.902440].

[42] M. Ahmed and G. Blackwood, "Astrophysics Decadal Survey 2020: Management Plan for Large Mission Concept Studies - Rev A,” 15 March 2016 (accessed 16 June 2016), http://science.nasa.gov/media/medialibrary/ 2016/03/15/Decadal_Studies_Management_Plan-RevA1_2016_03_15.pdf. 